Article

\title{
Predictor-Based Motion Tracking Control for Cloud Robotic Systems with Delayed Measurements
}

\author{
Shaobo Shen ${ }^{1} \mathbb{D}$, Aiguo Song ${ }^{1, * \mathbb{C}}$ and Tao $\mathrm{Li}^{2}$ \\ 1 School of Instrument Science and Engineering, Southeast University, Nanjing 210096, China; \\ shenshaobo@live.cn \\ 2 School of Automation Engineering, Nanjing University of Aeronautics and Astronautics, \\ Nanjing 210016, China; autolitao@nuaa.edu.cn \\ * Correspondence: a.g.song@seu.edu.cn
}

Received: 18 February 2019; Accepted: 1 April 2019; Published: 3 April 2019

\begin{abstract}
This paper addresses the problem of motion prediction and tracking control for cloud robotic systems with time-varying delays in measurements. A novel method using an observer-based structure for position and velocity prediction is developed to estimate the real-time information of robot manipulator. The prediction error can converge to zero even if model uncertainties exist in the robot manipulator. Based on the predicted positions and velocities, some sufficient conditions are derived to design suitable tracking controllers such that semi-globally uniformly ultimately bounded tracking performance of the predictor-controller couple can be guaranteed. Finally, the effectiveness and robustness to model uncertainties of the proposed method are verified by a two degree-of-freedom (DOF) robot system.
\end{abstract}

Keywords: motion prediction; nonlinear predictor; robotic control; time delay; tracking control

\section{Introduction}

Nowadays, with the development of Internet and communication technologies, cloud robotics combining cloud computing with robotics have become an attractive research topic in the recent years [1-3]. As a consequence, remote control of robot systems can be performed in a cloud platform through data connection with a robot manipulator. Important applications of cloud robotics can be found in space exploration, remote surgery, intelligent housing systems, unmanned vehicles, and so on [4-9].

Due to remote communication between robot sensors and control platforms, time delays are inevitable in measurement channels of a robotic system [10-12]. As such, real-time information of robot manipulator cannot arrive at the cloud platform on time, which may break down the operational capability or even destabilize the robotic system [13-15]. Thus, time delays in measurement channels are a non-negligible issue [16,17], and several approaches have been proposed to deal with the delayed measurements [18]. For instance, in [19], a dynamically smooth controller based on linear matrix inequality (LMI) techniques was studied to guarantee asymptotic tracking of a robot system subject to delayed measurements. In [20], a position feedback controller for Internet-based telerobotic systems with time delays was proposed using the Lyapunov-Krasovskii functional method [21]. However, these works focus mainly on the stability issue of controller designs. The delayed information of position measurements of the robot was still used in the controller, which results in a delayed tracking performance of the robot system. Thus, a prediction method that can provide more precise real-time motion feedback from robots to control platforms and human operators is very essential.

Recently, various prediction methods have been developed for linear robotic systems with delayed measurements. For examples, a Smith predictor approach was proposed to estimate robot models [22]. 
An optimal estimation approach for continuous-time systems was carried out based on Kalman filtering using delayed measurements in [23]; and a linear predictor was designed for a mobile robot in [24]. Nevertheless, for nonlinear systems, especially for the Euler-Lagrange system, few results taking delayed measurements into account are available. In recent years, some observer-based methods were proposed to predict actual states using delayed outputs. In [25], an observer-based predictor was proposed under LMI conditions to estimate the position and force based on time-delayed data. In [26], interval observers for linear time-delay systems were devised to obtain guaranteed interval estimate of system states. In $[27,28]$, the cascade observers (predictors) were utilized for the long constant time delay. However, accurate mathematical models of systems were required in some existing literature, which restricts the application area of the proposed methods. In addition, in [25-28], the controller design problem was not considered.

As for the problem of global motion tracking for nonlinear robot systems with an observercontroller structure, in [29], global uniform asymptotic stability was studied under an output feedback controller by employing nonlinear cascaded system theory. In [30], asymptotic stability of the observer-controller system was guaranteed through the Lyapunov functional approach. However, time delays were not considered in those works. Moreover, the nondelayed measurements of joint positions rather than the observation results were directly used in the controllers. Hence, such approaches cannot be applied to the case of delayed measurements. To the authors' best knowledge, few tracking controller design methods have been reported using only the predictions for the Euler-Lagrange system.

Motivated by the work in [31], where a tracking controller design method was presented for the Euler-Lagrange system with constant input delay, this paper proposes a novel observer-based prediction method for an $n$-DOF telerobotic system with a time-varying delay in position measurements. Both positions and velocities can be estimated through a simple model-independent predictor, which has a proportion-integration-differentiation(PID)-like regulator for delayed prediction error. It is proven that the prediction error can converge to zero by applying the Lyapunov method. The proposed predictor can also be put into use independently of the controller, such that the cloud platform can feed back more precise real-time messages of robots to the human operator. Moreover, as an important part of the proposed control framework, a tracking controller based on both predicted positions and velocities is designed such that the closed-loop system can achieve semi-globally uniformly ultimately bounded tracking. Finally, simulation is made through a two-DOF robot system to demonstrate the effectiveness of the proposed results.

The main contributions of this paper lie in two aspects. First, we propose a model-independent predictor-controller structure for motion tracking control of robotic systems subject to measurement (output) delay, which is different from [31-33], where some control design methods are presented for nonlinear systems with input delays. Second, we design suitable tracking controller based on prediction information about positions and velocities rather than based on non-delayed measurements as done in $[29,30]$. Therefore, the proposed method in this paper is more appropriate for applications of cloud robotics compared with the ones in $[29,30]$.

This paper is organized as follows. In the next section, some preliminaries and problem formulation are given. The motion prediction method is presented in Section 3. A tracking controller is designed in Section 4. Simulation results are shown in Section 5. Conclusions and some future works are discussed in Section 6.

\section{Problem Statement}

Consider a class of uncertain nonlinear $n$-DOF robot system represented by

$$
M(q) \ddot{q}+C(q, \dot{q}) \dot{q}+F(q, \dot{q})=u-f_{e}
$$


where $q \in \mathbb{R}^{n}$ is the vector of the joint positions, $\dot{q}$ is the the vector of the joint velocities, $u \in \mathbb{R}^{n}$ is the control input, $f_{e} \in \mathbb{R}^{n}$ is the unknown environment force generated by objective target, $F(q, \dot{q}) \in \mathbb{R}^{n \times n}$ is an unknown function which represents unmodeled dynamics of robot manipulator, $M(q) \in \mathbb{R}^{n \times n}$ is the positive definite inertia matrix, and $C_{m}(q, \dot{q}) \in \mathbb{R}^{n \times n}$ is the matrix of the centripetal and coriolis torque, which satisfy the following structural property of robotic systems:

Property 1: The inertia matrix $M(q)$ is definitely positive. There exist the positive constants $m_{1}$ and $m_{2}$ such that $m_{1} I \leq M(q) \leq m_{2} I$, where $I$ is an identity matrix.

Property 2: The matrix $\dot{M}(q)-2 C(q, \dot{q})$ is skew symmetric.

Property 3: There exists a positive scalar $c$ such that $\|C(q, \dot{q})\| \leq c\|\dot{q}\|$.

Our first main objective is to predict the actual state of system (1) by using the delayed position measurements:

$$
y_{d}=q(t-\tau(t)),
$$

where $\tau(t)$ is a time-varying delay. Then, the control objective is tracking a desired trajectory $q_{d}(t) \in \mathbb{R}^{n}$, which is given by the human operator. The proposed control structure of the cloud robotic system is shown in Figure 1.

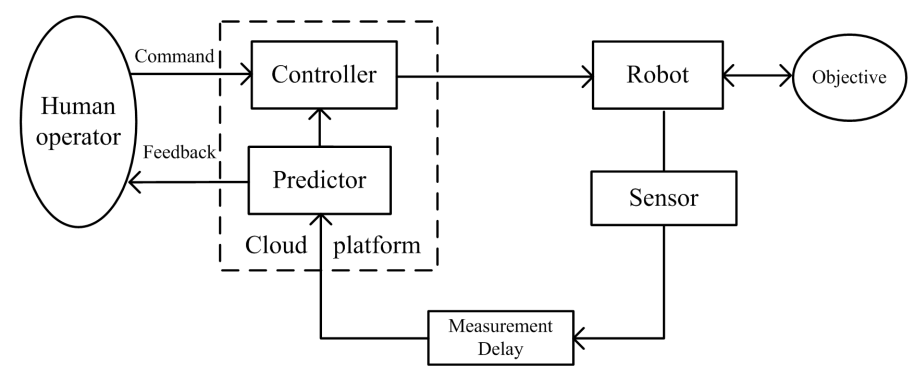

Figure 1. Control structure of cloud robot system with delayed measurement.

To facilitate the subsequent design, we rewrite the system (1) as

$$
\begin{aligned}
& \dot{x}_{1}(t)=x_{2}(t), \\
& \dot{x}_{2}(t)=f\left(x_{1}, x_{2}, u, t\right)+S_{1}(t),
\end{aligned}
$$

where $x_{1}=q, x_{2}=\dot{q}, x=\left[\begin{array}{ll}x_{1}^{T} & x_{2}^{T}\end{array}\right]^{T}$, and

$$
\begin{aligned}
& f\left(x_{1}, x_{2}, u, t\right)=M^{-1}\left(x_{1}\right)\left(-C\left(x_{1}, x_{2}\right) \dot{x}_{2}+u\right), \\
& S_{1}(t)=M^{-1}\left(x_{1}\right)\left(-F\left(x_{1}, x_{2}\right)-f_{e}\right) .
\end{aligned}
$$

The predictor and controller design will be carried out under the following usual assumptions:

Assumption 1: The desired trajectory $x_{d}=q_{d} \in \mathbb{R}^{n}$ is designed such that the $i-t h(i=1,2,3)$ time derivative of $x_{d}$ exist and are bounded by known positive constants.

Assumption 2: $f\left(x_{1}, x_{2}, u, t\right)$ is an $\ell^{2}$ function.

Assumption 3: The time-varying delay $\tau(t)$ is bounded and satisfies [34-36]

$$
0 \leq \tau(t) \leq \tau_{0} .
$$

Assumption 4: The unknown part $S_{1}(t)$ with its time derivative are bounded functions and satisfy

$$
\left\|S_{1}\right\| \leq d_{1},\left\|\dot{S}_{1}\right\| \leq d_{2}
$$


Remark 1. As explained and illustrated in [33,37], these assumptions are quite common in the framework of state observation of nonlinear time delay systems. It will be shown in the following analysis that the uncertain part $S_{1}(t)$ will not determine the global asymptotic convergence of prediction errors. However, we also find in our simulations that a more accurate model can make a faster convergence rate.

\section{Predictor Design}

\subsection{Predictor Formulation}

Let us denote the prediction errors as

$$
\begin{aligned}
& e_{1}=x_{1}-\hat{x}_{1}, \\
& e_{2}=\dot{e}_{1}+\alpha e_{1},
\end{aligned}
$$

where $\hat{x}_{1}, \hat{x}_{2}$ represent the prediction results of $x_{1}, x_{2}$, respectively, and $\alpha$ is a positive constant.

The proposed prediction method with an observer-based structure is given as

$$
\begin{aligned}
& \dot{\hat{x}}_{1}(t)=\hat{x}_{2}(t), \\
& \dot{\hat{x}}_{2}(t)=f\left(\hat{x}_{1}, \hat{x}_{2}, u, t\right)+\varphi(t-\tau(t)),
\end{aligned}
$$

with

$$
\begin{aligned}
\varphi(t) & =K\left(e_{2}(t)+\beta \int_{t_{0}}^{t} e_{2}(\sigma) d \sigma\right)+K_{0} \Pi(t)+K \int_{t_{0}}^{t}(\varphi(\theta-\tau(t))-\varphi(\theta)) d \theta, \\
\dot{\Pi}(t) & =\operatorname{Sgn}(\eta(t)),
\end{aligned}
$$

where $K=K_{a}+K_{b}$ and $K_{0}$ represent the predictor gains, $\beta$ is a positive constant, and $\operatorname{Sgn}(\cdot)$ represents the standard signum function. Let

$$
e_{\varphi}(t)=\varphi(t-\tau(t))-\varphi(t)
$$

Then, the auxiliary error $\eta \in \mathbb{R}^{n}$ is defined as

$$
\eta=e_{2}+e_{\varphi f}
$$

where $e_{\varphi f} \in \mathbb{R}^{n}$ is a filtered version of $e_{\varphi}$, i.e.,

$$
\dot{e}_{\varphi f}=-\beta e_{\varphi f}+e_{\varphi}
$$

Remark 2. It should be noticed that the proposed predictor (12) has a PID structure for the delayed prediction error $e_{1}(t-\tau(t))=x_{1}(t-\tau(t))-\hat{x}_{1}(t-\tau(t))$, which means that the prediction results $\hat{x}_{1}$ and $\hat{x}_{2}$ should be reserved for later use of the predictor.

To facilitate the subsequent analysis, an auxiliary observation error $r(t) \in \mathbb{R}^{n}$ is defined as

$$
r(t)=\dot{\eta}(t)+\beta \eta(t)=\dot{e}_{2}(t)+\beta e_{2}(t)+e_{\varphi}(t) .
$$

It can be obtained that

$$
\dot{\varphi}(t)=K r(t)+K_{0} \operatorname{Sgn}(\eta) .
$$

Using the system functions (3)-(4) and definitions (9)-(10), we have

$$
\begin{aligned}
r & =\dot{x}_{2}-\dot{\hat{x}}_{2}+\alpha \dot{e}_{1}+\beta e_{2}+\dot{\hat{x}}_{2}-f\left(\hat{x}_{1}, \hat{x}_{2}, u, t\right)-\varphi \\
& =S_{1}+S_{2}-\varphi,
\end{aligned}
$$


where $S_{1}$ has been defined in (6) and $S_{2} \in \mathbb{R}^{n}$ is given as

$$
S_{2}=f\left(x_{1}, x_{2}, u, t\right)-f\left(\hat{x}_{1}, \hat{x}_{2}, u, t\right)+\alpha \dot{e}_{1}+\beta e_{2} .
$$

The time derivative of (20) can be obtained as follows:

$$
\dot{r}(t)=N_{d}+N-e_{2}(t)-K r(t)-K_{0} \operatorname{Sgn}(\eta),
$$

where

$$
\begin{aligned}
N_{d} & =\dot{S}_{1}, \\
N & =\dot{S}_{2}+e_{2}(t) .
\end{aligned}
$$

The Mean Value Theorem can be utilized to find an upper bound for $N$ as [32]:

$$
\|N\| \leq \rho(\| z||)|| z \|,
$$

where $\|\cdot\|$ denotes the standard Euclidean norm, $\rho(\cdot)$ is a positive and non-decreasing function, and $z \in \mathbb{R}^{4 n}$ is defined as

$$
z=\left[\begin{array}{llll}
e_{1}^{T} & e_{2}^{T} & e_{\varphi}^{T} & r^{T}
\end{array}\right]^{T} .
$$

Then, based on Assumption 3, we can deduce

$$
\left\|N_{d}\right\| \leq d_{2} .
$$

\subsection{Prediction Error Analysis}

Theorem 1. The predictor described by Equations (11) and (12) ensures semi-global asymptotic regulation of $e_{1}$ and $e_{2}$ in the sense that $e_{1} \rightarrow 0$ and $e_{2} \rightarrow 0$ as $t \rightarrow \infty$, provided that the matrix $K_{a}$ are selected sufficiently large relative to the system initial conditions and the following conditions are simultaneously satisfied:

$$
\begin{array}{r}
\alpha-\frac{1}{2}>0, \\
\beta-\frac{1}{2}-\frac{\delta}{2}>0, \\
\frac{1}{\tau_{0}}-1-\frac{1}{2 \delta}>0, \\
K_{b}>0, \\
K_{0}>\left\|N_{d}\right\|_{\infty}+\left\|\dot{N}_{d}\right\|_{\infty},
\end{array}
$$

where $\delta$ is a positive constant, and $\|\cdot\|_{\infty}$ denotes the $\ell_{\infty}$ norm.

Proof of Theorem 1. Let $y(t) \in \mathbb{R}^{3 n+1}$ be defined as

$$
y=\left[\begin{array}{lllll}
e_{1}^{T} & e_{2}^{T} & r^{T} & \sqrt{Q} & \sqrt{P}
\end{array}\right]^{T},
$$

where $Q(t) \in \mathbb{R}$, and $P(t) \in \mathbb{R}$ are selected as

$$
\begin{aligned}
& Q=\int_{t-\tau_{0}}^{t} \int_{s}^{t}\|\dot{\varphi}(\vartheta)\|^{2} d \theta d s, \\
& P=\xi-\int_{t_{0}}^{t} r(\theta)\left[N_{d}(\theta)-K_{0} \operatorname{Sgn}(\eta(\theta))\right] d \theta .
\end{aligned}
$$


The non-negativeness of $P(t)$ has been proved in [32,37], provided that the gain $K_{0}$ satisfies inequality (32) and $\xi$ is selected as:

$$
\xi=\sum_{i=1}^{n} K_{0 i}|| e_{i}(0) \|-e^{T}(0) N_{d}(0),
$$

where the subscript $i$ denotes the $i$ th element of the vector or diagonal matrix.

Let $V(y, t) \in \mathbb{R}$ be a Lyapunov functional which is defined as follows:

$$
V=\frac{1}{2} e_{1}^{T} e_{1}+\frac{1}{2} e_{2}^{T} e_{2}+\frac{1}{2} r^{T} r+Q+P
$$

and it can be bounded as

$$
\frac{1}{2}\|y\|^{2} \leq V(y, t) \leq\|y\|^{2}
$$

After taking the time derivative of $V$, we have

$$
\begin{aligned}
\dot{V}= & e_{1}^{T}\left(e_{2}-\alpha e_{1}\right)+e_{2}^{T}\left(r-\beta e_{2}-e_{\varphi}\right)+r^{T}\left(N+N_{d}-e_{2}-K r\right)+\dot{Q}+\dot{P} \\
= & -\alpha\left\|e_{1}\right\|^{2}-\beta\left\|e_{2}\right\|^{2}+e_{1} e_{2}-e_{2} e_{\varphi}+r^{T} N-r^{T} K r \\
& +\tau_{0}\|\dot{\varphi}(t)\|^{2}-\int_{t-\tau_{0}}^{t}\|\dot{\varphi}(\theta)\|^{2} d \theta .
\end{aligned}
$$

Using Young's inequality, we have

$$
\begin{aligned}
\left\|e_{2}^{T} e_{\varphi}\right\| & \leq \frac{\delta}{2}\left\|e_{2}\right\|^{2}+\frac{1}{2 \delta}\left\|e_{\varphi}\right\|^{2}, \\
\left\|e_{1}^{T} e_{2}\right\| & \leq \frac{1}{2}\left\|e_{1}\right\|^{2}+\frac{1}{2}\left\|e_{2}\right\|^{2} .
\end{aligned}
$$

Then, we use Jensen's inequality to derive

$$
\begin{aligned}
& \left\|e_{\varphi}\right\|^{2} \leq \tau_{0} \int_{t-\tau_{0}}^{t}\|\dot{\varphi}(\theta)\|^{2} d \theta, \\
& \|\dot{\varphi}(t)\|^{2} \leq \int_{t-\tau_{0}}^{t}\|\dot{\varphi}(\theta)\|^{2} d \theta .
\end{aligned}
$$

Substituting the inequalities (40)-(43) into (39), the upper bound of $\dot{V}$ can be obtained as

$$
\begin{aligned}
\dot{V} \leq & -\left(\alpha-\frac{1}{2}\right)\left\|e_{1}\right\|^{2}-\left(\beta-\frac{1}{2}-\frac{\delta}{2}\right)\left\|e_{2}\right\|^{2}+\|r\| \rho(\|z\|)\|z\|-K r^{T} r \\
& -\frac{1}{\tau_{0}}\left(1-\tau_{0}-\frac{\tau_{0}}{2 \delta}\right)\left\|e_{\varphi}\right\|^{2} .
\end{aligned}
$$

Then, after completing the squares by using the definition of $\|z\|$ in (26), the inequality in (44) can be bounded as

$$
\dot{V} \leq-\left[\omega-\frac{\rho^{2}(\|z\|)}{4 K_{a}}\right]\|z\|^{2}
$$

where $\omega \in \mathbb{R}$ is defined as

$$
\omega=\min \left\{\alpha-\frac{1}{2}, \beta-\frac{1}{2}-\frac{\delta}{2}, \frac{1}{\tau_{0}}-1-\frac{1}{2 \delta}, K_{b}\right\} .
$$


Thus, the negative semi-definiteness of $\dot{V}(t)$ can be ensured once

$$
K_{a} \geq \frac{\rho^{2}(|| z||)}{4 \omega} \geq \frac{\rho^{2}\left(|| z_{0} \|\right)}{4 \omega}
$$

where $\left\|z_{0}\right\|$ is the initial condition of $\|z\|$.

Since $\dot{V}(t) \equiv 0$ means $e_{1} \equiv 0, e_{2} \equiv 0$, and $e_{\varphi} \equiv 0$, according to the definitions in Equations (34) and (35), we have $\sqrt{P} \equiv 0$ and $\sqrt{Q} \equiv 0$. By using LaSalle's invariance theorem, we have

$$
\begin{aligned}
& \lim _{t \rightarrow \infty} e_{1}=0, \\
& \lim _{t \rightarrow \infty} e_{2}=0 .
\end{aligned}
$$

This completes the proof of Theorem 1.

\section{Controller Development}

In this section, we consider the tracking controller design problem for the robotic system using the estimated positions and velocities. Let the tracking errors be denoted as

$$
\begin{aligned}
& \varepsilon_{0}=x_{d}-x, \\
& s_{0}=\dot{\varepsilon}_{0}+\alpha \varepsilon_{0} .
\end{aligned}
$$

Because of the time delay in measurement channel, $\varepsilon_{0}$ and $s_{0}$ cannot be used for the controller design. The auxiliary errors are respectively defined as

$$
\begin{aligned}
& \varepsilon=x_{d}-\hat{x}_{1}=\varepsilon_{0}+e_{1}, \\
& s=\dot{\varepsilon}+\alpha \varepsilon=\varepsilon_{0}+\alpha \dot{\varepsilon}_{0}+e_{2}=s_{0}+e_{2} .
\end{aligned}
$$

Utilizing the system model (1) and the definition of tracking error (51), we can obtain the dynamics of $s_{0}$ as

$$
\begin{gathered}
M(q) \dot{s}_{0}=-C(q, \dot{q}) s_{0}+Y \theta-u+f_{e}+F(q, \dot{q}), \\
Y \theta=M(q)\left(\ddot{q}_{d}+\alpha \dot{\varepsilon}_{0}\right)+C(q, \dot{q})\left(\dot{q}_{d}+\alpha \varepsilon_{0}\right) .
\end{gathered}
$$

The proposed tracking controller is given as

$$
u=K_{u} s=K_{u} s_{0}+K_{u} e_{2},
$$

where $K_{u}=K_{c}+K_{d}$ represents the controller gain. The block diagram of proposed predictor-controller structure is shown in Figure 2.

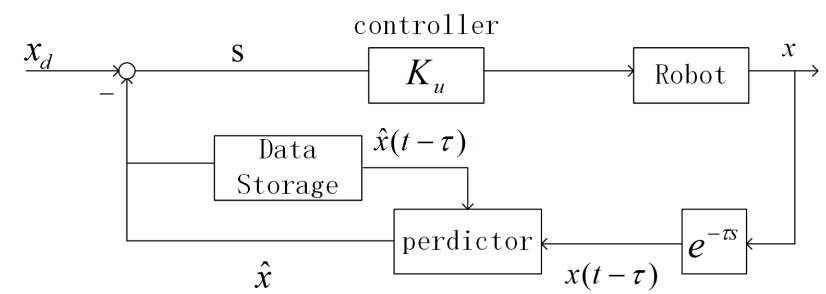

Figure 2. Block diagram of proposed predictor-controller structure. 
Theorem 2. The predictors of (11)-(12) and the control law in (56) ensure semi-globally uniformly ultimately bounded tracking for the system (1) in the sense that

$$
\left\|\varepsilon_{0}(t)\right\| \leq \varepsilon_{0}(0) e^{-\gamma t}+\frac{d}{\gamma}
$$

provided that the matrix $K_{c}$ are selected sufficiently large relative to the system initial conditions and the following conditions are simultaneously satisfied:

$$
\begin{array}{r}
\alpha-\frac{1}{2}>0, \\
\beta-\frac{1}{2}-\frac{\delta}{2}-\frac{1}{2 \delta}>0, \\
\frac{1}{\tau_{0}}-1-\frac{1}{2 \delta}-\frac{1}{\delta^{2}}>0, \\
K_{b}>0, K_{d}>0, \\
K_{0}>\left\|N_{d}\right\|_{\infty}+\left\|\dot{N}_{d}\right\|_{\infty},
\end{array}
$$

where $\gamma, d \in \mathbb{R}^{+}$denote constants.

Proof of Theorem 2. Let $Y_{d} \theta \in \mathbb{R}^{n}$ be defined as

$$
Y_{d} \theta=M\left(q_{d}\right) \ddot{q}_{d}+C\left(q_{d}, \dot{q}_{d}\right) \dot{q}_{d}
$$

According to Assumption $1, Y_{d} \theta$ can be bounded as

$$
\left\|Y_{d} \theta\right\| \leq d_{\theta}
$$

Similar to the upper bound of $\mathrm{N}$ in (25), we can use the Mean Value Theorem to obtain that

$$
\left\|Y \theta-Y_{d} \theta\right\| \leq \rho_{v}(\|v\|)\|v\|
$$

where $\rho_{v}(\cdot)$ is a positive known bounding and nondecreasing function. Moreover, $v(t) \in \mathbb{R}^{2 n}$ is defined as

$$
v=\left\{\varepsilon_{0}^{T} s_{0}^{T}\right\}^{T}
$$

Then, the Equation (54) can be rewritten as

$$
M(q) \dot{s}_{0}=-C(q, \dot{q}) s_{0}+Y \theta-Y_{d} \theta-u+f_{e}+Y_{d} \theta+F(q, \dot{q})
$$

Consider following Lyapunov functional defined as

$$
V_{c}=\frac{1}{2} s_{0}^{T} M(q) s_{0}+\frac{1}{2} \varepsilon_{0}^{T} \varepsilon_{0}+\dot{V}
$$

Acording to Property 1, we have

$$
l_{1}\left\|y_{c}\right\|^{2} \leq V_{c} \leq l_{2}\left\|y_{c}\right\|^{2}
$$

where $l_{1}=\min \left\{\frac{1}{2}, m_{1}\right\}, l_{2}=\min \left\{1, m_{2}\right\}$, and

$$
y_{c}=\left[\begin{array}{ll}
v^{T} & y^{T}
\end{array}\right]^{T} .
$$


Taking the time derivative of the Lyapunov functional in (68) and using the Property 2 result in

$$
\begin{gathered}
\dot{V}_{c} \leq|| s_{0}\left\|\rho_{v}(\|v\|)\right\| v\left\|-K_{u}\right\| s_{0}\left\|^{2}-K_{u}\right\| s_{0} \mid\|\| e_{2} \| \\
+d_{m}\left\|s_{0}\right\|+\left\|s_{0}\right\|\left\|\varepsilon_{0}\right\|-\alpha\left\|\varepsilon_{0}\right\|^{2}+\dot{V}
\end{gathered}
$$

where $d_{m}=\frac{d_{1}}{m_{1}}+d_{\theta}$. Using the Young's inequalities

$$
\begin{aligned}
& \left\|s _ { 0 } \left|\left\||| e_{2}\right\| \leq \frac{\delta}{2}\left\|s_{0}\right\|^{2}+\frac{1}{2 \delta}\left\|e_{2}\right\|^{2},\right.\right. \\
& \left\|s _ { 0 } \left|\left\||| \varepsilon_{0}\right\| \leq \frac{1}{2}\left\|s_{0}\right\|^{2}+\frac{1}{2}\left\|\varepsilon_{0}\right\|^{2},\right.\right.
\end{aligned}
$$

we have

$$
\begin{gathered}
\dot{V}_{c} \leq \\
\left\|s_{0}\right\| \rho_{v}(\|v\|)\|v\|-\left(K_{u}+\frac{1}{2}\left(\delta K_{u}-1\right)\right)\left\|s_{0}\right\|^{2} \\
-\left(\alpha-\frac{1}{2}\right)\left\|\varepsilon_{0}\right\|^{2}+\frac{1}{2 \delta}\left\|e_{2}\right\|^{2}+d_{m}\left\|s_{0}\right\|+\dot{V} .
\end{gathered}
$$

After substituting $\dot{V}$ of (44) and completing the squares, the upper bound of inequality (74) can be determined as

$$
\begin{aligned}
\dot{V}_{c} \leq & -\left[\lambda_{1}-\frac{\rho_{v}^{2}(\|v\|)}{4 K_{c}}\right]\|v\|^{2}+\frac{d_{m}^{2}}{4 K_{d}} \\
& -\left[\lambda_{2}-\frac{\rho^{2}(\|z\|)}{4 K_{a}}\right]\|z\|^{2}-\frac{\tau}{\delta^{2}} \int_{t-\tau}^{t}\|\dot{\varphi}(\theta)\|^{2} d \theta,
\end{aligned}
$$

where

$$
\begin{gathered}
\lambda_{1}=\min \left\{\frac{1}{2}\left(\delta K_{u}-1\right), \alpha-\frac{1}{2}\right\}, \\
\lambda_{2}=\min \left\{\alpha-\frac{1}{2}, \beta-\frac{1}{2}-\frac{\delta}{2}-\frac{1}{2 \delta}, \frac{1}{\tau_{0}}-1-\frac{1}{2 \delta}-\frac{1}{\delta^{2}}, K_{b}\right\} .
\end{gathered}
$$

Using the definitions of $z$ in (26), $y$ in (33), $y_{c}$ in (70), and the following inequality

$$
Q=\int_{t-\tau_{0}}^{t} \int_{s}^{t}\|\dot{\varphi}(\theta)\|^{2} d \theta d s \leq \tau_{0} \int_{t-\tau_{0}}^{t}\|\dot{\varphi}(\theta)\|^{2} d \theta
$$

we can obtain

$$
\dot{V}_{c} \leq-\gamma\left\|y_{c}\right\|^{2}+d=-\gamma V_{c}+d,
$$

where

$$
\begin{gathered}
d=\frac{d_{m}^{2}}{4 K_{d}}+\frac{\xi}{\delta^{2}} \\
\gamma=\min \left\{\lambda_{1}-\frac{\rho_{v}^{2}(\|v\| \mid)}{4 K_{c}}, \lambda_{2}-\frac{\rho^{2}(\| z||)}{4 K_{a}}, \frac{1}{\delta^{2}}\right\},
\end{gathered}
$$

and $\gamma \in \mathbb{R}$ is a positive constant, provided that

$$
K_{a} \geq \frac{\rho^{2}(\|z\|)}{4 \lambda_{2}}, K_{c} \geq \frac{\rho^{2}(\|v\|)}{4 \lambda_{1}} .
$$

Then, we have

$$
V_{c}(y, t) \leq V_{c}(0) e^{-\gamma t}+\frac{d}{\gamma}
$$


Together with $V_{c}$ in (68), the tracking error $\varepsilon_{0}$ converges to the bounded region exponentially and its bound is determined by $d$. This completes the proof of Theorem 2 .

Remark 3. Since $\rho(\cdot)$ and $\rho_{v}(\cdot)$ are positive nondecreasing functions and $\|z\|,\|v\|$ are decreasing, the inequalities in (82) are equivalent to

$$
K_{a} \geq \frac{\rho^{2}\left(\left\|z_{0}\right\|\right)}{4 \lambda_{2}}, K_{c} \geq \frac{\rho^{2}\left(\left\|v_{0}\right\|\right)}{4 \lambda_{1}}
$$

where $z_{0}$ and $v_{0}$ are initial conditions of $z$ and $v$, respectively.

Remark 4. Since $\varepsilon_{0}$ is unavailable for the controller due to the measurement delay, the uncertain term $d_{1}\left\|s_{0}\right\|$ in (71) can not be compensated by using $\operatorname{Sgn}\left(\varepsilon_{0}\right)$. On the other hand, in the uncertainty-free case $\left(d_{1}=d_{2}=0\right)$, the compensation function $\Pi(t)$ in predictors (11)-(12) and $P(t)$ in Lyapunov functional (37) can be omitted. In addition, the controller can be designed as $u=-Y_{d} \theta+K_{u}$ s to compensate for the term $Y \theta$ and eliminate $d_{\theta}$ as in [30]. Then, from (83), we can obtain that $d=0$ and the tracking error converges to 0 in the uncertainty-free case.

\section{Simulations}

In this section, simulations are presented to verify the effectiveness of the proposed method including position prediction and tracking control. We consider a two-DOF robot system which is shown in Figure 3 , where $l_{1}, l_{2}, m_{1}, m_{2}$ is the lengths and weights of robot arms, respectively. The inertia matrix as well as the centripetal and coriolis matrix are given as

$$
M(q)=\left[\begin{array}{ll}
M_{11} & M_{12} \\
M_{21} & M_{22}
\end{array}\right], C(q, \dot{q})=\left[\begin{array}{ll}
C_{11} & C_{12} \\
C_{21} & C_{22}
\end{array}\right],
$$

with

$$
\begin{aligned}
M_{11} & =\left(2 l_{1} \cos q_{2}+l_{2}\right) l_{2} m_{2}+l_{1}^{2}\left(m_{1}+m_{2}\right), M_{12}=M_{21}=l_{2}^{2} m_{2}+l_{1} l_{2} m_{2} \cos q_{2}, M_{22}=l_{2}^{2} m_{2}, \\
C_{11} & =-l_{1} l_{2} m_{2} \sin \left(q_{2}\right) \dot{q}_{2}, C_{12}=-l_{1} l_{2} m_{2} \sin \left(q_{2}\right)\left(\dot{q}_{1}+\dot{q}_{2}\right), C_{21}=l_{1} l_{2} m_{2} \sin \left(q_{2}\right) \dot{q}_{1}, C_{22}=0 .
\end{aligned}
$$

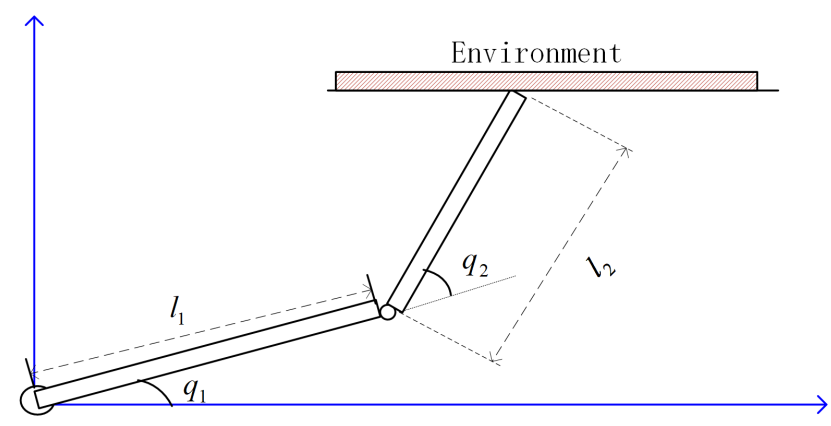

Figure 3. The 2-DOF robot.

To verify the robustness to system uncertainties of our method, we assume that the mathematical model of robot manipulator is entirely unknown in the simulations. The matrices $M(q)$ and $C(q, \dot{q})$ are unknown for the designer. Thus, we additionally treat $C(q, \dot{q}) \dot{q}$ and the input $u$ as the uncertain part when we rewrite the system model into the form of functions (3) and (4). That is, $f\left(x_{1}, x_{2}, u, t\right)=0$ and $S_{1}=M^{-1}\left(x_{1}\right)\left[-C_{m}\left(x_{1}, x_{2}\right) x_{2}+u-f_{e}\right]$.

The desired position trajectories are given as $q_{r 1}=0.2 \sin (0.15 t)$ and $q_{r 2}=0.1 \sin (0.15 t)$ and an environment force $f_{e}=0.01 \dot{q}+0.05 \sin (q)$ is applied to the robot. The previously mentioned 
parameters and control gains are given in Table 1 . Then, we consider the time-varying measurement delay which is randomly varying from $0-0.5 \mathrm{~s}$ as shown in Figure 4.

Table 1. Parameters.

\begin{tabular}{cccccccc}
\hline $\boldsymbol{m}_{\mathbf{1}}$ & $\boldsymbol{m}_{\mathbf{2}}$ & $\boldsymbol{l}_{\mathbf{1}}$ & $\boldsymbol{l}_{\mathbf{2}}$ & $\boldsymbol{K}$ & $\boldsymbol{K}_{\boldsymbol{u}}$ & $\boldsymbol{\alpha}$ & $\boldsymbol{\beta}$ \\
\hline $1 \mathrm{~kg}$ & $0.5 \mathrm{~kg}$ & $0.5 \mathrm{~m}$ & $0.5 \mathrm{~m}$ & 25 & 0.1 & 5 & 10 \\
\hline
\end{tabular}

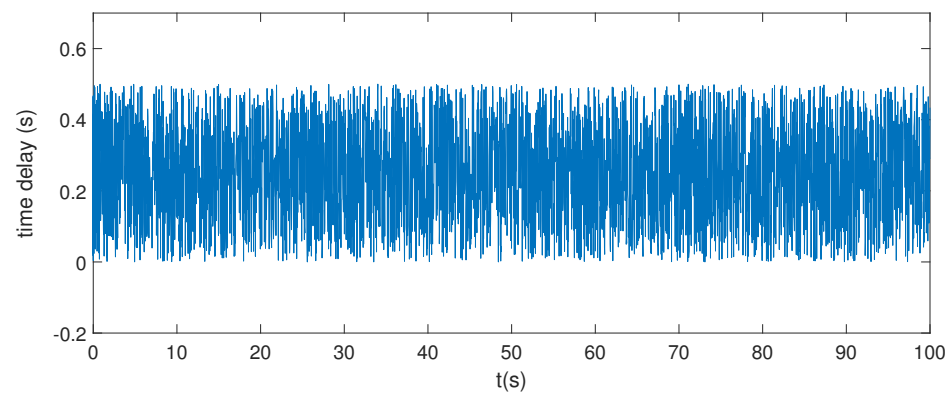

Figure 4. Time-varying delay.

Firstly, the prediction results of positions and velocities of the proposed method are shown in Figures 5 and 6, respectively. The real values of each joint positions and velocities are labeled as $q 1, q_{2}$ and $v 1, v_{2}$, while the predicted ones are labeled as $q 1 e, q_{2} e$ and $v 1 e, v_{2} e$, respectively. It can be seen that the elegant prediction performance of proposed observer-based predictor is achieved. Both predicted positions and velocities can converge to the real ones. Meanwhile, the position tracking results are given in Figure 7, where the desired positions are labeled as $q r 1$ and $q r 2$. Moreover, the proposed controller is compared with the one without predictor (using the delayed output directly). The position tracking errors are shown in Figure 8. The tracking performance and robustness to model uncertainties of proposed controller can be proved.

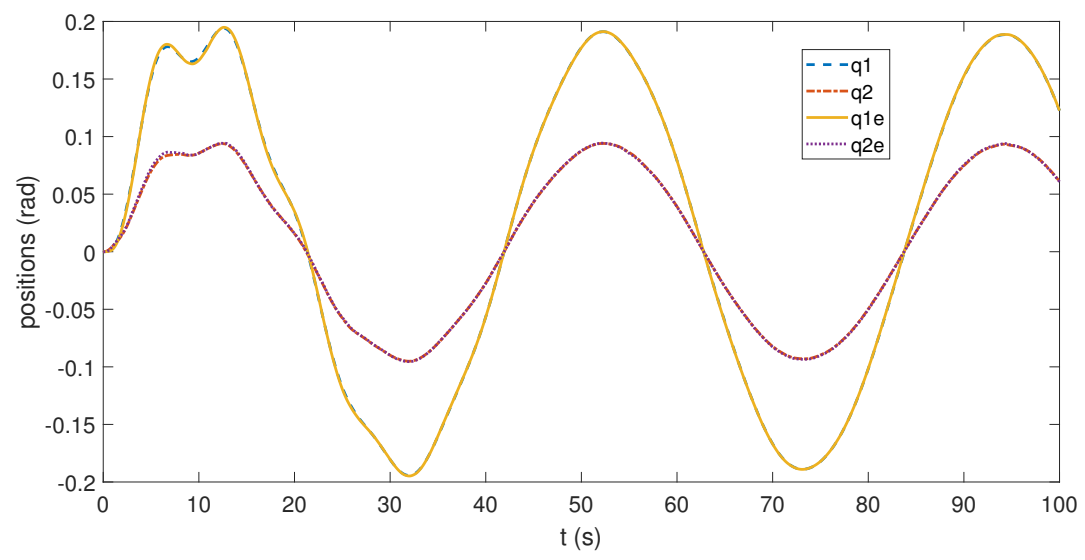

Figure 5. Position predictions under time-varying delay.

Secondly, the proposed predictor of our work is compared with the high gain predictor utilized in [27], which can be represented as

$$
\dot{\hat{x}}(t)=f(\hat{x}, u, t)+K^{\prime}(x(t-\tau(t))-\hat{x}(t-\tau(t)),
$$

where the predictor gain $K^{\prime}=3$ is used in our simulation. The compared results of prediction errors of positions and velocities are shown in Figures 9 and 10, respectively. It can be seen that, because of the PID structure of our methods, the proposed predictor has faster convergence abilities of both position 
and velocity prediction errors than the high gain predictor does. Although the convergence rate can be improved by a larger predictor gain $K^{\prime}$, the stability of a high gain predictor will be destroyed when $K^{\prime}>5$ in our simulations. In addition, the controller design method using predictor (85) has not been discussed in [27]. In our simulations, the instability has been shown when we combine the high gain predictor with our proposed controller, which can also indicate better capability and stronger robustness of the proposed predictor in this paper.

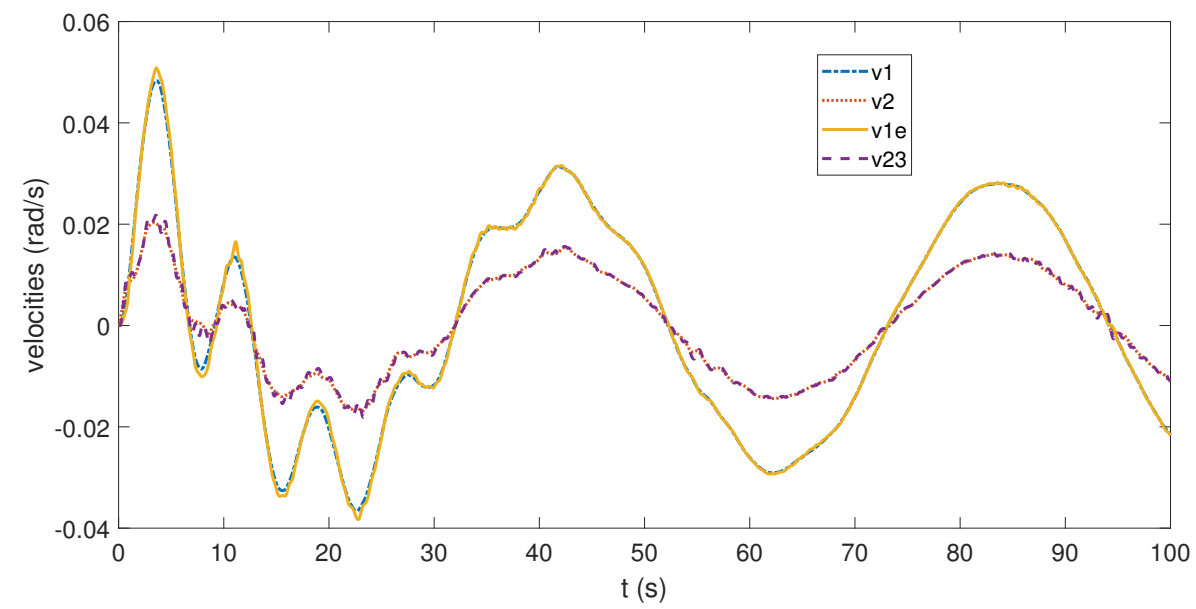

Figure 6. Velocity predictions under time-varying delay.

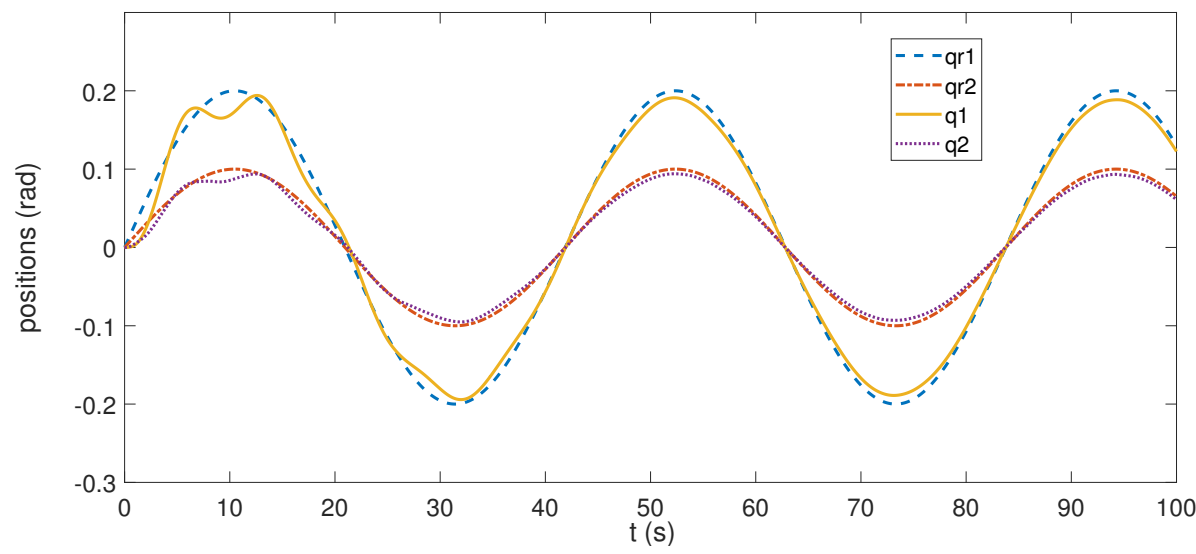

Figure 7. Position tracking performance under time-varying delay.

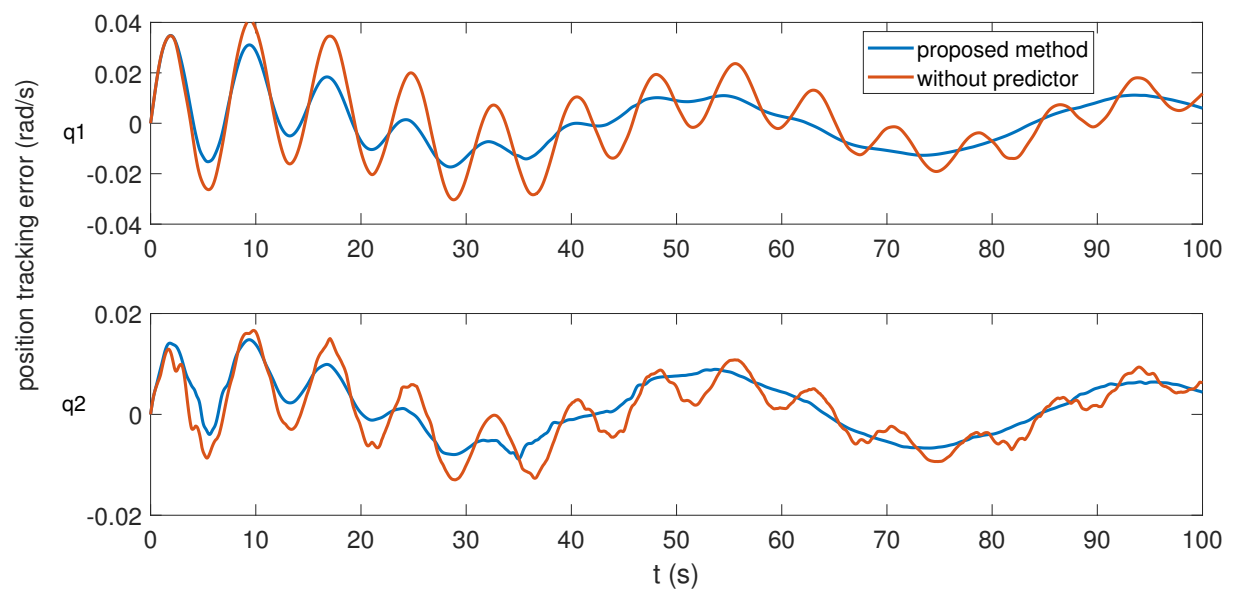

Figure 8. Compared results of position tracking errors. 

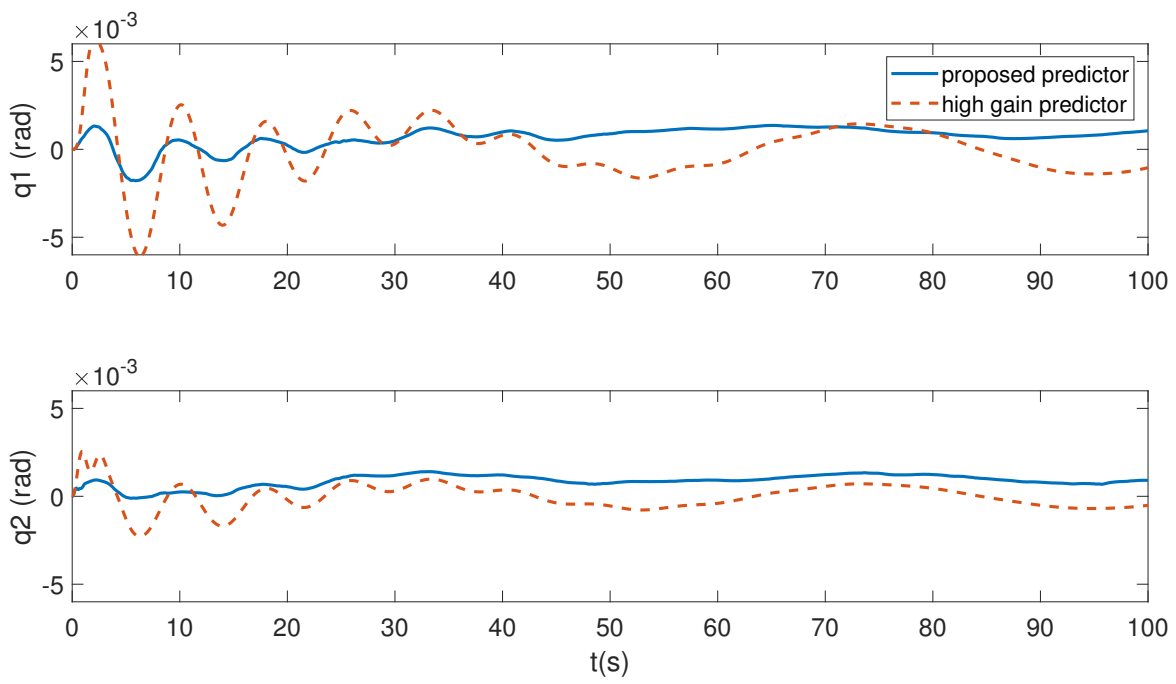

Figure 9. Compared results of position prediction errors.
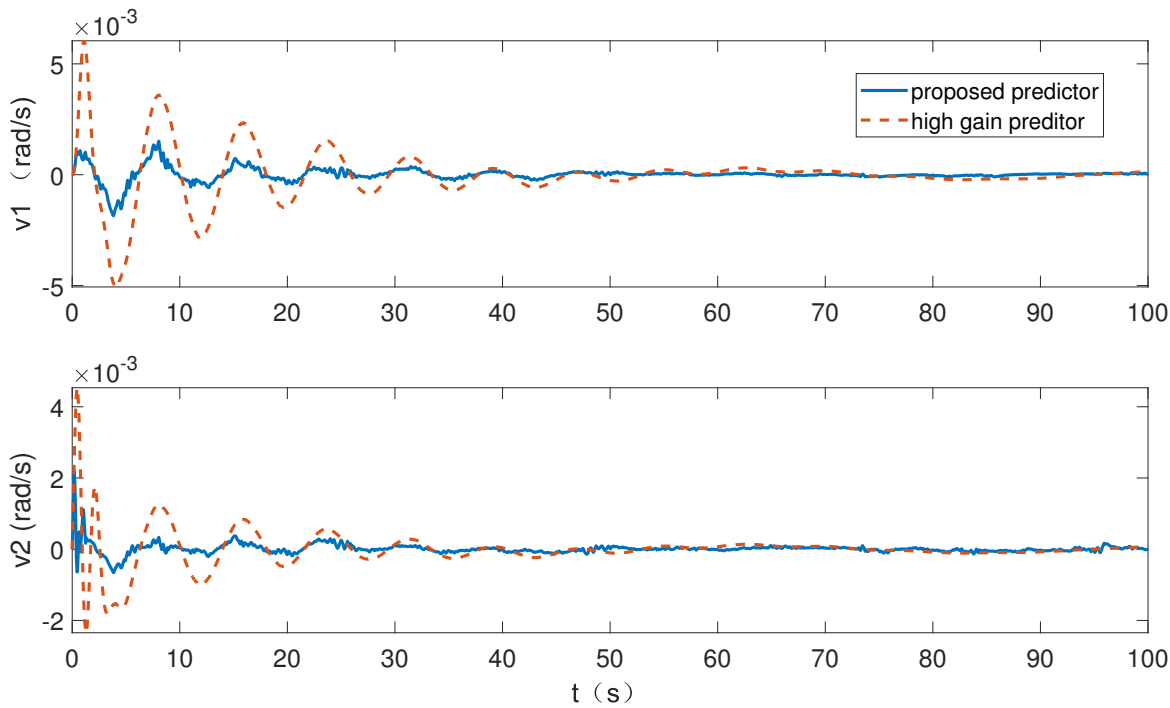

Figure 10. Compared results of velocity prediction errors.

\section{Conclusions}

In this paper, a novel observer-based motion predictor has been developed for cloud robotic systems with measurement delay. Both positions and velocities can be estimated though a simple model-independent predictor. Then, a tracking controller using both predicted positions and velocities has also been designed. The semi-global stability of both prediction and tracking errors has been proved using the Lyapunov direct method. The measurement delay can be effectively compensated by using the predictions. Simulation results have shown the good performance and strong robustness to time-varying delays and model uncertainties of the proposed method. Further studies will be focused on the advanced robust controller design with predictions for some nonlinear systems [38,39].

Author Contributions: Conceptualization, S.S. and A.S.; methodology, S.S. and T.L.; software, S.S.; validation, S.S, A.S. and T.L.; formal analysis, T.L.; investigation, S.S.; resources, A.S.; data curation, S.S.; writing-original draft preparation, S.S. and T.L.; writing - review and editing, A.S.; visualization, S.S.; supervision, A.S.; project administration, A.S.; funding acquisition, A.S. 
Funding: This work was supported by the National Key Research and Development Program of China under Grant No. 2016YFB1001301, and the National Natural Science Foundation of China under Grant No. 91648206 and No. U1713210.

Conflicts of Interest: The authors declare no conflict of interest.

\section{References}

1. Chen Y.; Du, Z.; Garca-aacosta, M. Robot as a service in cloud computing. In Proceedings of the Fifth IEEE International Symposium on Service Oriented System Engineering, Nanjing, China, 4-5 June 2010; pp. 151-158.

2. Ramírez De La Pinta, J.; Maestre Torreblanca, J.M.; Jurado, I.; Reyes De Cozar, S. Off the shelf cloud robotics for the smart home: Empowering a wireless robot through cloud computing. Sensors 2017, 17, 525. [CrossRef]

3. Vega, J.; Cañas, J.M. PiBot: An open low-cost robotic platform with camera for STEM education. Electronics 2018, 7, 430. [CrossRef]

4. Al-Wais, S.; Mohajerpoor, R.; Shanmugam, L.; Abdi, H.; Nahavandi, S. Improved delay-dependent stability criteria for telerobotic systems with time-varying delays. IEEE Trans. Syst. Man Cybern. Syst. 2017, 48, 2470-2484. [CrossRef]

5. Sugiura, K.; Shiga, Y.; Kawai, H.; Misu, T. A cloud robotics approach towards dialogue-oriented robot speech. Adv. Robot. 2015, 29, 449-456. [CrossRef]

6. Jahanshahi, H.; Jafarzadeh, M.; Sari, N.N.; Pham, V.-T.; Huynh, V.V.; Nguyen, X.Q. Robot motion planning in an unknown environment with danger space. Electronics 2019, 8, 201. [CrossRef]

7. Ding, L.; Han, Q.L.; Zhang, X. Distributed secondary control for active power sharing and frequency regulation in islanded microgrids using an event-triggered communication mechanism. IEEE Trans. Ind. Inform. 2018. [CrossRef]

8. Rabah, M.; Rohan, A.; Han, Y.-J.; Kim, S.-H. Design of fuzzy-PID controller for quadcopter trajectory-tracking. Int. J. Fuzzy Logic Intell. Syst. 2018, 18, 204-213. [CrossRef]

9. Tiep, D.K.; Lee, K.; Im, D.-Y.; Kwak, B.; Ryoo, Y.-J. Design of fuzzy-PID controller for path tracking of mobile robot with differential drive. Int. J. Fuzzy Logic Intell. Syst. 2018, 18, 220-228. [CrossRef]

10. Shahbazi, M.; Atashzar, S.F.; Tavakoli, M.; Patel, R.V. Position-force domain passivity of the human arm in telerobotic systems. IEEE/ASME Trans. Mechatron. 2018, 23, 552-562. [CrossRef]

11. Santos, J.; Conceição, A.; Santos, T.; Araújo, H. Remote control of an omnidirectional mobile robot with time-varying delay and noise attenuation. Mechatronics 2018, 52, 7-21. [CrossRef]

12. Li, W.; Liang, D.; Gao, H.; Tavakoli, M. Haptic tele-driving of wheeled mobile robots under nonideal wheel rolling, kinematic control and communication time delay. IEEE Trans. Syst. Man Cybern. Syst. 2017, 99, 1-12. [CrossRef]

13. Farooq, U.; Gu, J.; El-Hawary, M.E.; Asad, M.U.; Abbas, G.; Luo, J. A time-delayed multi-master-single-slave non-linear tele-robotic system through state convergence. IEEE Access 2018, 6, 5447-5459. [CrossRef]

14. Nuno, E.; Basanez, L.; Ortega, R. Passivity-based control for bilateral teleoperation: A tutorial. Automatica 2011, 47, 485-495. [CrossRef]

15. Lee, J.; Chang, P.H.; Jin, M. Adaptive integral sliding mode control with time-delay estimation for robot manipulators. IEEE Trans. Ind. Electron. 2017, 64, 6796-6804. [CrossRef]

16. Zhang, X.; Han, Q.; Ge, X.; Ding, D. An overview of recent developments in Lyapunov-Krasovskii functionals and stability criteria for recurrent neural networks with time-varying delays. Neurocomputing 2018, 313, 392-401. [CrossRef]

17. Zhang, X.; Han, Q.; Wang, Z.; Zhang, B. Neuronal state estimation for neural networks with two additive time-varying delay components. IEEE Trans. Cybern. 2017, 47, 3184-3194. [CrossRef]

18. Lian, H.; Xiao, S.; Wang, Z.; Zhang, X.; Xiao, H. Further results on sampled-data synchronization control for chaotic neural networks with actuator saturation. Neurocomputing 2019. [CrossRef]

19. Yang, C.; Wang, X.; Li, Z.; Li, Y.; Su, C.-Y. Teleoperation control based on combination of wave variable and neural networks. IEEE Trans. Syst. Man Cybern. Syst. 2017, 47, 2125-2136. [CrossRef]

20. Hua, C.; Liu, X.P. Teleoperation over the internet with/without velocity signal. IEEE Trans. Instrum. Meas. 2010, 60, 4-13. [CrossRef]

21. Zhang, X.; Han, Q.; Seuret, A.; Gouaisbaut, F.; He, Y. Overview of recent advances in stability of linear systems with time-varying delays. IET Control Theory Appl. 2019, 13, 1-16. [CrossRef] 
22. Bowthorpe, M.; Tavakoli, M.; Becher, H.; Howe, R. Smith predictor-based robot control for ultrasound-guided teleoperated beating-heart surgery. IEEE J. Biomed. Health Inform. 2014, 18, 157-166. [CrossRef]

23. Zhang, H.; Lu, X.; Cheng, D. Optimal estimation for continuous-time systems with delayed measurements. IEEE Trans. Autom. Control 2006, 51, 823-827. [CrossRef]

24. Guechi, E.H.; Lauber, J.; Dambrine, M.; Defoort, M. Output feedback controller design of a unicycle-type mobile robot with delayed measurements. IET Control Theory Appl. 2012, 6, 726-733. [CrossRef]

25. Lu, Z.; Huang, P.; Liu, Z. Predictive approach for sensorless bimanual teleoperation under random time delays with adaptive fuzzy control. IEEE Trans. Ind. Electron. 2018, 65, 2439-2448. [CrossRef]

26. Efimov, D.; Fridman, E.; Polyakov, A.; Perruquetti, W.; Richard, J.-P. Linear interval observers under delayed measurements and delay-dependent positivity. Automatica 2016, 72, 123-130. [CrossRef]

27. Ahmed-Ali, T.; Cherrier, E.; Lamnabhi-Lagarrigue, F. Cascade high gain predictors for a class of nonlinear systems. IEEE Trans. Autom. Control 2014, 57, 221-226. [CrossRef]

28. Hernández, O.; Farza, M.; Ménard, T.; Targui, B.; M'Saad, M.; Astorga-Zaragoza, C.M. A cascade observer for a class of MIMO non uniformly observable systems with delayed sampled outputs. Syst. Control Lett. 2016, 98, 86-96. [CrossRef]

29. Borhaug, E.; Pettersen, K.Y. Global output feedback PID control for n-DOF Euler-Lagrange systems. In Proceedings of the 2006 American Control Conference, Minneapolis, MN, USA, 14-16 June 2006; pp. 4993-4999.

30. Erkan, Z.; Enver, T.; Egemen, K. A model independent observer based output feedback tracking controller for robotic manipulators with dynamical uncertainties. Robotica 2017, 35, 729-743.

31. Sharma, N.; Bhasin, S.; Wang, Q.; Dixon, W.E. Predictor-based control for an uncertain Euler-Lagrange system with input delay. Automatica 2011, 47, 2332-2342. [CrossRef]

32. Obuz, S.; Tatlicioglu, E.; Cekic, S.C.; Dawson, D.M. Predictor-based robust control of uncertain nonlinear systems subject to input delay. IFAC Proc. Vol. 2012, 45, 231-236. [CrossRef]

33. Kamalapurkar, R.; Fischer, N.; Obuz, S.; Dixon, W.E. Time-varying input and state delay compensation for uncertain nonlinear systems. IEEE Trans. Autom. Control 2015, 61, 834-839. [CrossRef]

34. Zhang, X.; Han, Q.; Zeng, Z. Hierarchical type stability criteria for delayed neural networks via canonical Bessel-Legendre inequalities. IEEE Trans. Cybern. 2018, 48, 1660-1671. [CrossRef] [PubMed]

35. Wang, J.; Zhang, X.; Han, Q. Event-triggered generalized dissipativity filtering for neural networks with time-varying delays. IEEE Trans. Neural Netw. Learn. Syst. 2016, 27, 77-88. [CrossRef] [PubMed]

36. Zhang, B.; Han, Q.; Zhang, X.; Yu, X. Sliding mode control with mixed current and delayed states for offshore steel jacket platform. IEEE Trans. Control Syst. Technol. 2014, 22, 1769-1783. [CrossRef]

37. Su, Y.; Muller, P.C.; Zheng, C. A Simple Nonlinear observer for a class of uncertain mechanical systems. IEEE Trans. Autom. Control 2007, 52, 1340-1345.

38. Zhou, L.; She, J.; Zhou, S.; Li, C. Compensation for state-dependent nonlinearity in a modified repetitive-control system. Int. J. Robust Nonlinear Control 2018, 28, 213-226. [CrossRef]

39. Zhou, L.; She, J.; Zhou, S. Robust $H_{\infty}$ control of an observer-based repetitive-control system. J. Frankl. Inst. 2018, 355, 4952-4969. [CrossRef]

(C) 2019 by the authors. Licensee MDPI, Basel, Switzerland. This article is an open access article distributed under the terms and conditions of the Creative Commons Attribution (CC BY) license (http://creativecommons.org/licenses/by/4.0/). 